\title{
Solvent Mixing To Induce Molecular Motor Aggregation into Bowl- Shaped Particles: Underlying Mechanism, Particle Nature, and Application To Control Motor Behavior
}

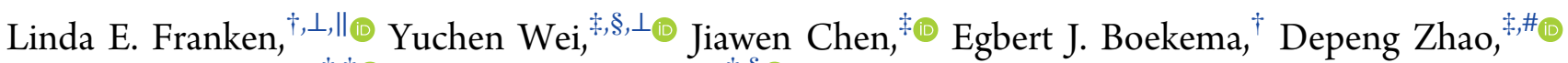 \\ Marc C. A. Stuart, $*,+\dagger$ and Ben L. Feringa $*, \$, \S(0)$
}

\begin{abstract}
${ }^{\dagger}$ Electron Microscopy Group, Groningen Biomolecular Sciences and Biotechnology Institute, University of Groningen, Nijenborgh 7, 9747 AG Groningen, The Netherlands

${ }^{\ddagger}$ Centre for Systems Chemistry, Stratingh Institute for Chemistry, University of Groningen, Nijenborgh 4, 9747 AG Groningen, The Netherlands

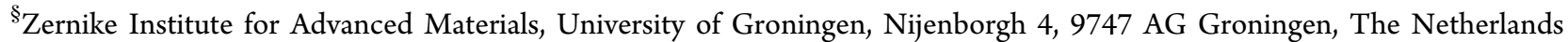

Supporting Information

\begin{abstract}
Control over dynamic functions in larger assemblies is key to many molecular systems, ranging from responsive materials to molecular machines. Here we report a molecular motor that forms bowl-shaped particles in water and how confinement of the molecular motor affects rotary motion. Studying the aggregation process in a broader context, we provide evidence that, in the case of bowl-shaped particles, the structures are not the product of self-assembly, but a direct result of the mixing a good solvent and a (partial) non-solvent and highly independent of the molecular design. Under the influence of the non-solvent, droplets are formed, of which the exterior is hardened due to the increase in the glass transition temperature by the external medium, while the interior of the droplets remains plasticized by the solvent, resulting in the formation of stable bowl-shaped particles with a fluid interior, a glass-like exterior, and a very specific shape: dense spheres with a hole in their side. Applying this to a bulky first-generation molecular motor allowed us to change its isomerization behavior. Furthermore, the motor shows in situ photo-switchable aggregation-induced emission. Strong confinement prohibits the thermal helix inversion step while altering the energy barriers that determine the rotary motion, such that it introduces a reverse trans-cis isomerization upon heating. These studies show a remarkable control of forward and backward rotary motion by simply changing solvent ratios and extent of confinement.
\end{abstract}

\section{INTRODUCTION}

The design of functional small molecules that can assemble into larger dynamic structures such as gels, vesicles, or nanocapsules has undergone rapid advances in recent years. $^{1-4}$ Illustrative are the development of new functional systems such as responsive ${ }^{5-8}$ and self-healing materials, ${ }^{9-12}$ nanocarriers, ${ }^{13-16}$ catalysts in confined space, ${ }^{17-19}$ and artificial muscles. ${ }^{20,21} \mathrm{~A}$ variety of structures have been introduced with increasing control over properties such as morphology, ${ }^{22-24}$ (dis)assembly, ${ }^{25}$ rheology, ${ }^{26,27}$ orthogonality, $^{28}$ and size. ${ }^{29}$

As part of our studies on molecular rotary motors in dynamic molecular systems, we address the challenge how rotary motors will operate in confined space in aqueous media. In this context, one particular morphology has drawn our attention, as it is both very specific in its shape and very general in its occurrence. Its nanosize structure comprises a dense, spherical aggregate with a small portion of material-a holemissing from the surface. This morphology has been coined hollow spheres, ${ }^{30-32}$ dimple-like aggregates, ${ }^{33}$ dimpled beads, ${ }^{34}$ cup-like aggregates, ${ }^{35}$ and bowl-shaped par- ticles. $^{32,36,37}$ We avoid introducing yet another name and use the term bowl-shaped particles. These aggregates have been indicated as (large compound) micelle ${ }^{38-41}$ or vesicle. ${ }^{31,42-48}$ Because of the peculiar hole makes that the structure is easily mistaken for a (collapsed) vesicle. When only scanning electron microscopy (SEM) is used without transmission electron microscopy (TEM), the two morphologies cannot be distinguished. ${ }^{32}$

Although this morphology is as specific as a double membrane layer, it is found in connection with a very wide range of molecules: amphiphiles, ${ }^{36,42,44}$ pseudo-amphiphiles, ${ }^{47}$ hydrophobic molecules, ${ }^{30,40}$ block copolymers, ${ }^{31,35-38,41,43,45,46}$ and many others structures. ${ }^{33,34,39}$ Yet the fundamental principle behind the formation of these bowlshaped aggregates, the method of solvent mixing, and the understanding of the morphology have, to our knowledge, not yet been elucidated.

Received: March 19, 2018

Published: June 7, 2018 
We discovered that the novel molecular motor 1 (Figure 1, Supporting Information (SI), Figures S1-S4) can aggregate

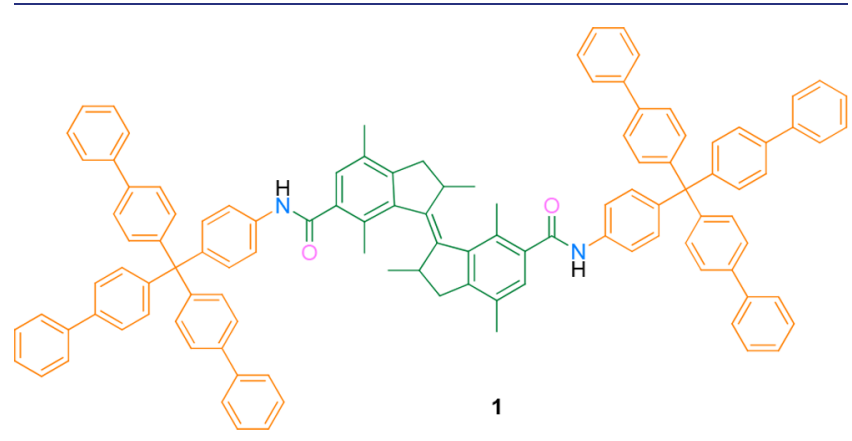

Figure 1. Chemical structure of molecular motor 1. The molecular motor contains a rotary core (green) and two bulky aromatic groups (orange), linked by amide groups.

into bowl-shaped particles in water and that their size and thereby molecular motor confinement can be controlled. This allows us to study the rotary behavior of molecular motor $\mathbf{1}$ in confined space and provides a unique way to control forward or backward rotary motion. These findings also allow us to address in a broader context some of the fundamental issues regarding the formation of bowl-shaped aggregates.

Careful inspection of all studies that obtained bowl-shaped particles led to the observation that the most commonly used method is the induction of aggregation by the mixing of solvents. ${ }^{34-39,43-46}$ Tuning self-assembly of amphiphilic block copolymers into various morphologies by use of selective solvent mixtures has been successfully shown. ${ }^{24,49-52}$ Typically, the amphiphilic or hydrophobic molecule is dissolved in a solvent favoring the hydrophobic components of the molecule, followed by addition of a selective (non-)solvent, such as water, to induce aggregation.

At this stage we consider it appropriate to refer to selfassembly being defined as processes that involve pre-existing components (separate or distinct parts of a disordered structure), are reversible, and can be controlled by proper design of the components. "Self-assembly" is thus not synonymous with "formation". 53 Applying this to the various compounds that show bowl-shaped morphologies in water, it appears that the molecular design of the components is not the controlling factor in the assembly. Instead, the aggregation is most probably mainly solvent driven, and we use here, besides motor $\mathbf{1}$, some other non-amphiphilic molecules to shed light on the mechanism of this bowl-shaped aggregation. Furthermore, our cryo-TEM images contradict a hollow nature of the bowl-shaped spheres.

In order to explore the nature of the small-molecule nanoaggregates and the potential of the bowl-shaped morphologies, we applied solvent mixing and our novel molecular motor $\mathbf{1}$ as a model system. Compound $\mathbf{1}$ belongs to a unique class of light-responsive molecules which are able to undergo $360^{\circ}$ unidirectional rotation. ${ }^{54-56}$ Powered by light, the central carbon-carbon double bond undergoes trans-cis isomerization, followed by the energetically downhill process of thermal helix inversion (THI). These photochemical and thermal steps induce a rotation by $180^{\circ}$ of one half of the motor relative to the other. By repetition, continuous unidirectional rotary motion is achieved. Importantly, the rotary direction is dictated by the methyl group(s) at the stereogenic center(s) next to the central double bond, which causes the enantiomers to display opposite rotary directions with respect to each other.

To date, most molecular motors have been studied in solutions or on surfaces, revealing that the surrounding environment, for example solvent viscosity, can affect the rotary motion of a molecular motor. ${ }^{57,58}$ Compared to solution systems, natural stimuli-responsive molecules such as photoresponsive peptides usually work in a more confined environment, where the isomerization processes can occur with enhanced selectivity. ${ }^{59,60}$ At the extreme, a complete solid state can have a major influence on the performance of many photo-responsive molecules. ${ }^{61-65}$ Here we shed light on the nature and formation of bowl-shaped particles and show the control of rotation of motor $\mathbf{1}$ due to aggregation in water into such bowl-shaped structures.

\section{RESULTS AND DISCUSSION}

Our design of molecular motor 1 comprises a first-generation light-driven motor core with two pending hydrophobic and rigid cone-shaped trisbiphenyl units linked via amide moieties (Figure 1; for synthesis and characterization, see SI, Figure S1, S2, S3, andS4; for isomerization processes, vide infra section 2.4).

2.1. Solvent Mixing with Various Molecules. Besides molecular motor 1, several hydrophobic molecules, i.e., polystyrene, Nile Red, styrofoam, and polyvinyl chloride, were initially tested to see if, in general, bowl-shaped particles can be obtained by first solubilizing the molecule in tetrahydrofuran (THF) and subsequently mixing the solution with water (Figures 2 and 3). After optimization of molecule

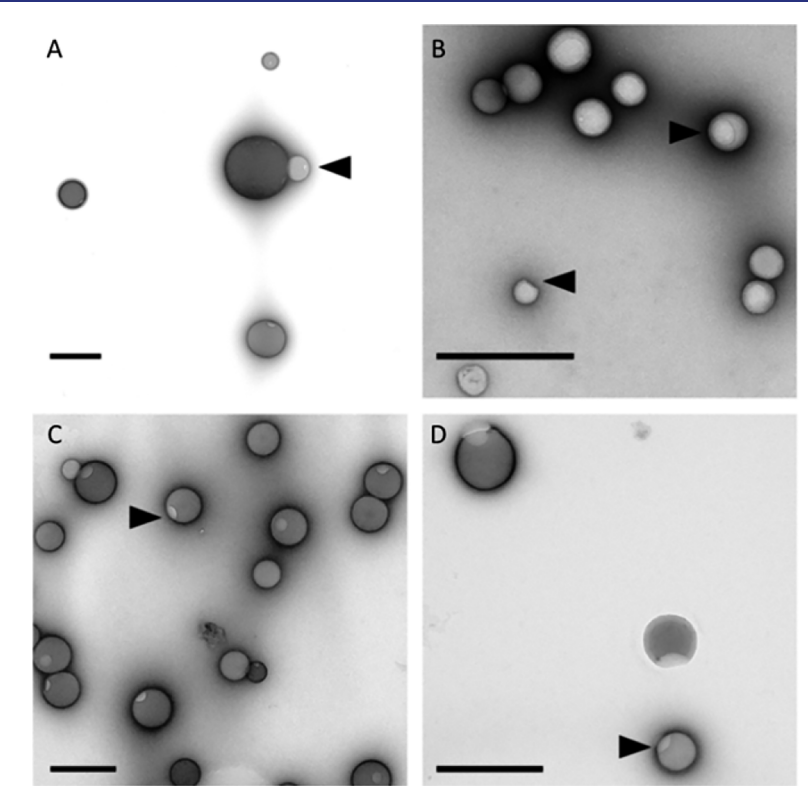

Figure 2. TEM images of bowl-shaped aggregates from several molecules stained with $2 \%$ uranyl acetate (UAc): (A) $1 \mathrm{mg} / \mathrm{mL}$ polystyrene PS174 in THF at $50 \% \varphi_{\mathrm{w}}$; (B) $0.5 \mathrm{mg} / \mathrm{mL}$ Nile Red at $75 \% \varphi_{\mathrm{w}} ;$ (C) $0.5 \mathrm{mg} / \mathrm{mL}$ Styrofoam in $50 \% \varphi_{\mathrm{w}} ;$ (D) $0.5 \mathrm{mg} / \mathrm{mL}$ polyvinyl chloride PVC17 at $66 \% \varphi_{\mathrm{w}}$. Reported are the starting concentration of the molecule in THF prior to mixing with water and the THF-water volume ratio after mixing, which were optimized for each sample to generate bowl-shaped particles. While samples A, C, and $\mathrm{D}$ had holes in the surface of nearly every particle, in B they were visible in only $20-40 \%$ of the particles. Scale bars represent $500 \mathrm{~nm}$, and arrows indicate examples of holes in the exterior. 
concentration and volume fraction of water $\left(\varphi_{\mathrm{w}}\right)$, the characteristic bowl-shaped particles were obtained through this method for all tested molecules. The bowl-shaped particles range roughly from 100 to $500 \mathrm{~nm}$ in size, and the majority has only one hole in its surface, although on rarer occasions multiple holes were observed.

In addition to THF-water, two other mixable solvents were tested: tert-butanol with water and chloroform with methanol (SI, Figure S5). Although particles were formed, the holes in the exterior were found more rarely, indicating an influence of solvent type on hole formation and/or size of bowl-shaped particles. ${ }^{30}$ Mixing of toluene and water has also been reported, ${ }^{30}$ but due to lack of miscibility, multiple steps are needed. Our results indicate that bowl-shaped particles can be obtained directly when solvents are used that are mixable, like THF in water.

2.2. Characterization of Bowl-Shaped Particles. The spheres from the rotary motor $\mathbf{1}$ were imaged using three different TEM preparation techniques: drying, negative staining, and cryo-TEM (Figure 3). ${ }^{66}$ Dense, not hollow, particles with holes are observed with all three techniques, demonstrating that the particles are stable, excluding a relationship between morphology and the TEM preparation and showing that particles are present in solution.

In order to test the stability over time, molecular motor 1 particles were prepared using $60 \%$ and $90 \% \varphi_{\mathrm{w}}$ and left for 4 days (Figure S6). At $90 \% \varphi_{\mathrm{w}}$, the particles remained unchanged in time (Figure S6D), while in the larger spheres that were created using only $60 \% \varphi_{\mathrm{w}}$, molecular motor 1 slowly crystallized in time (Figure S6B). Another distinct property of the bowl-shaped particles is their difference in size at different $\varphi_{\mathrm{w}}$. DLS data show that the particle size shrinks with increasing $\varphi_{\mathrm{w}}$. At $60 \% \varphi_{\mathrm{w}}$, a particle radius of $392 \mathrm{~nm}$ with a polydispersity of $64 \%$ is measured, whereas at $90 \% \varphi_{\mathrm{w}}$ the radius is $130 \mathrm{~nm}$ with a polydispersity of $35 \%$ (Figure $\mathrm{S} 7$ ). The shrinking/swelling of the aggregates is reversible by adding water or THF, respectively.

The stability of the mature bowl-shaped particles allowed us to wash them by pelleting and resuspension in $\mathrm{D}_{2} \mathrm{O}$ in order to remove all traces of THF and water from the surrounding
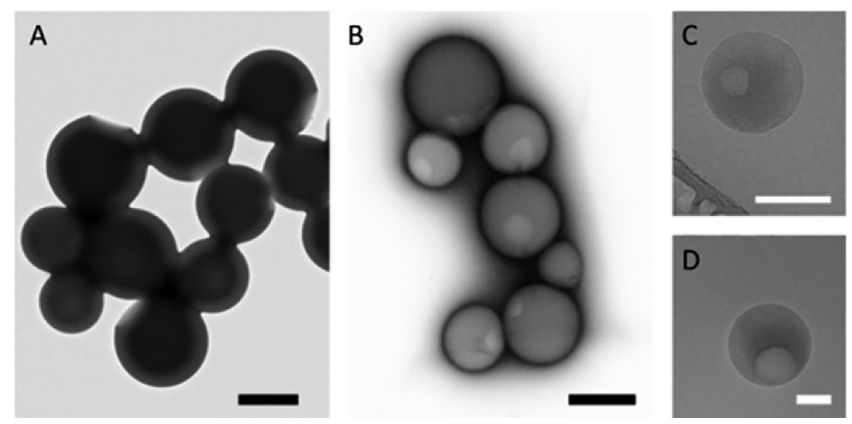

Figure 3. Spheres from the molecular motor 1 imaged by three TEM preparation techniques: drying (A), negative staining (B), and cryoTEM (C and D). Motor concentration was $10^{-4} \mathrm{M}$ in THF. Panels A and $\mathrm{B}$ are imaged at $60 \% \varphi_{\mathrm{w}}$ and $\mathrm{C}$ and $\mathrm{D}$ at $90 \% \varphi_{\mathrm{w}}$ for reasons of particle size. It should be noted that drying alone is not a proper preparation method for these systems, as the drying of droplets can also lead to bowl-shaped particles, ${ }^{31,33,38,47}$ and it cannot be distinguished whether the holes are formed upon drying or in solution. ${ }^{66}$ Scale bars represent $1 \mu \mathrm{m}$ (black) and $100 \mathrm{~nm}$ (white), respectively. medium prior to solubilizing the particles in $\mathrm{CDCl}_{3}$ for $\mathrm{NMR}$ (SI section 1.4). TEM observations confirm the unaffected nature of the spheres after washing, while NMR confirms the presence of THF in the spheres (Figure 4).

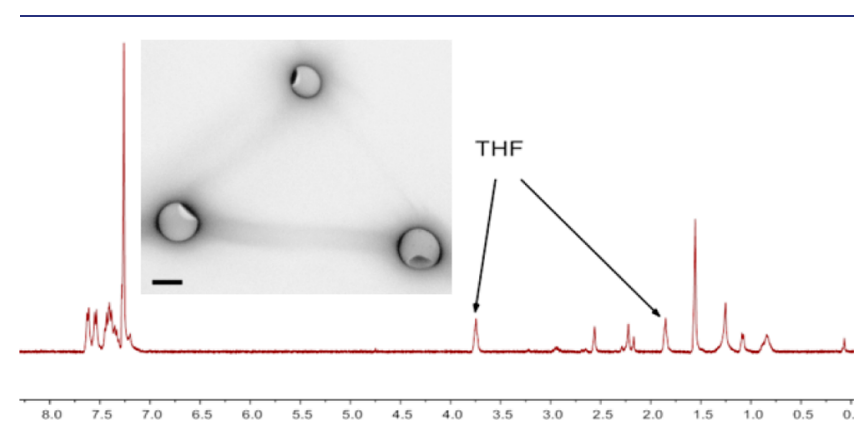

Figure 4. ${ }^{1} \mathrm{H}$ NMR spectrum $\left(\mathrm{CDCl}_{3}\right)$ of bowl-shaped particles from molecular motor 1 at $10^{-4} \mathrm{M}$ at $\varphi_{\mathrm{w}} 90 \%$ after excessive washing with $\mathrm{D}_{2} \mathrm{O}$. Insert: TEM image after washing the particles. Arrows point to absorptions corresponding to THF. Scale bar equals $200 \mathrm{~nm}$.

The practices of washing, stirring overnight, and dialysis to remove the initial solvent are commonly used. ${ }^{24,32,35-39,52}$ However, our experiments show that in our bowl-shaped aggregates, and likely in related systems, solvent remains inside the spheres, even after extensive washing.

2.3. Proposed Mechanism and Particle Nature. The driving forces behind the formation of bowl-shaped particles can be found in the field of amphiphilic block-copolymer selfassembly, in particular in the work of Eisenberg et al. ${ }^{24,36,49-52}$ The results with macromolecules show major consistency with our own observations with small molecules and identify the same parameters. ${ }^{36}$ In stark contrast to our findings stands the fact that the large majority of their systems (exempted ${ }^{36}$ ) display various morphologies in response to altered solvent ratios. In those systems, each block of the amphiphilic molecules responds differently to the solvent changes. The corresponding molecular reorganization is thus driven by selfassembly and dependent on molecular design. ${ }^{24,49-52}$ Besides self-assembly, Eisenberg et al. identify two key factors that govern the obtained morphologies: thermodynamics versus kinetics. ${ }^{24}$ As long as the thermodynamics of the molecular response to the changing medium is faster than the change in kinetics, the structures are in equilibrium before they become kinetically frozen by high water content. Kinetic freezing of a structure at a certain stage of reorganization is achieved by adding a large amount of selective solvent (non-solvent), ${ }^{36}$ which causes (part of) the molecular assembly to go below the glass transition temperature $\left(T_{\mathrm{g}}\right)^{52}$

This observation can be extrapolated to other systems, such as hydrophobic small molecules. Since hydrophobic molecules do not have partial but rather complete repulsion from the selective solvent, this non-solvent causes phase separation (Figure S8) but does not induce self-assembly. Bowl-shaped particles are formed when the spheres are kinetically frozen before self-assembly could take place. This can be due to a high $T_{\mathrm{g}}$ of the molecule in relation to the solvents or the slow (or missing) kinetics of the combined system.

The fact that non-amphiphilic molecules can form (bowlshaped) spheres seems distinct from descriptions such as "micellation", self-assembly, micelle, or large compound micelle (LCM). Those terms imply reorganization as a consequence of molecular properties which does not seem to 
be the driving force of bowl-shaped particles, where solvent is key. This is also true for several weak amphiphiles, which may be too slow to reach thermodynamic equilibrium and get kinetically frozen prior to self-assembly. ${ }^{24}$

We propose a mechanism of formation of bowl-shaped spheres as shown in Figure 5 that does not include self-

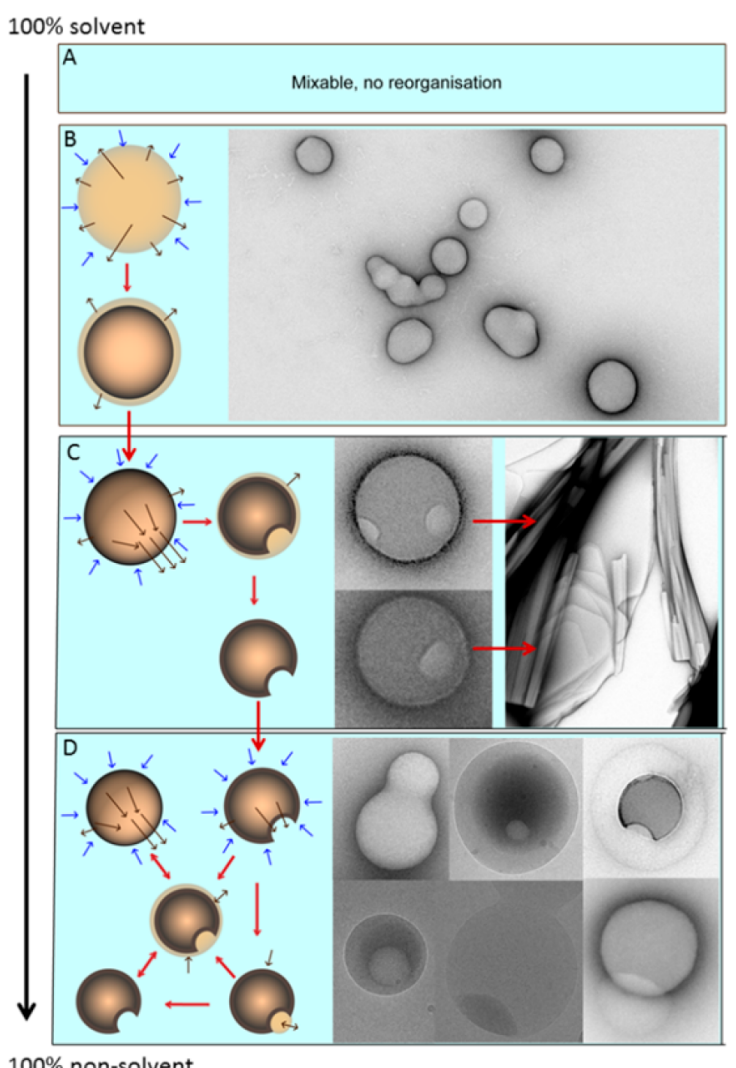

Figure 5. Schematized formation and sequence of morphologies upon increased amount of non-solvent. Up to a critical water content, the molecule remains soluble in the medium (A), after which phase separation into droplets occurs (B). The solvent at the exterior of the droplet mixes with the medium (black arrows), and the $T_{\mathrm{g}}$ of the exterior increases, leading to a hardened particle (glassy shell) (C). The shrinking particle builds pressure (blue arrows) against the plasticized solvent-containing interior, and the particle bursts at its weakest point, leaving a hole. While stable, over time these plasticized particles are still able to change. With more non-solvent, the particles keep shrinking, and the unfavorable medium causes the particles to hold on tighter to the solvent, leading to a balanced mixture of morphologies (D).

assembly and provides new insights into the nature of the spheres and their derivatives. Upon addition of a critical amount of selective solvent, initial aggregation of the material occurs into amorphous, unorganized droplets of the molecule with initial solvent. Their viscosity can vary depending on the properties of the initial solvent (plasticizer effect) ${ }^{67}$ and the starting concentration of the molecule. The selective solvent causes the shrinking of the exterior droplets by release of solvent to the water. Loss of solvent increases the particles' $T_{\mathrm{g}}$ and the exterior of the droplet hardens, whereas the inside remains fluid. The shrinkage causes continued release of solvent, but as the droplet exterior is less permeable, the hole in the particle is formed as the solvent bursts through the weakest part of the glass shell. At this stage, the balance between interior fluidity under plasticizer strength against increase of $T_{\mathrm{g}}$ due to the unfavorable medium allows the bowlshaped particles to still reorganize over longer time scales. At large amounts of selective solvent (e.g., $\mathrm{H}_{2} \mathrm{O}$ ), further compression squeezes solvent through the hole, which is the weakest part of the shell (Figure 5). In contrast to the method described by Im et al., ${ }^{30}$ this mechanism is highly dependent on the mixability of the two solvents, and the bowl-shaped particles that are generated are not necessarily solid due to the plasticizing effect of internal solvent. In fact increase of the $T_{\mathrm{g}}$ by the non-solvent leads to the solidification of the exterior. As the exterior shrinks faster than the interior, the pressure increases until the point that the shell bursts, leaving a hole in the side of the glass-like droplet (see also SI section 1.7 and Movie S1). The ready formation, the stability, the presence of solvents inside the bowl-shaped particles, and the reversibility of their size offer an excellent platform to study dynamic functions in confined space.

2.4. Control of Dynamic Function. First the rotary behavior of the bulky molecular motor 1 was investigated in THF solution. Figure $6 \mathrm{~A}$ shows the $360^{\circ}$ unidirectional rotary cycle typical for first-generation motors. This includes two photo-isomerization and two thermal isomerization steps. ${ }^{54,68}$ Upon irradiation with $312 \mathrm{~nm}$ UV light, trans-stable 1 undergoes a trans-cis isomerization, yielding a less stable isomer. This is indicated by the downfield shift of the aliphatic ring protons in the ${ }^{1} \mathrm{H}$ NMR spectra (Figure 6B). ${ }^{1} \mathrm{H}$ NMR shows an excellent photostationary state (PSS); the ratio between the two isomers is $95 \%$ cis-unstable 1 and 5\% transstable 1. Circular dichroism (CD) spectroscopy using enantiopure trans-stable $\mathbf{1}$ also confirms this light-triggered trans-cis isomerization process with concomitant helix inversion (the emergence of a new positive $\mathrm{CD}$ band at 350 nm, Figure 6C).

Conducting a subsequent THI by heating cis-unstable 1 at $50{ }^{\circ} \mathrm{C}$ for $12 \mathrm{~h}$ yields the stable cis-isomer, as confirmed by ${ }^{1} \mathrm{H}$ NMR (Figure 6B). Importantly, this thermal process is the rate-determining step, and its kinetics and thermodynamic parameters were investigated by UV/vis, which indicates the standard Gibbs energy of activation and the half-life of cisunstable 1 to be $102.2 \pm 4.2 \mathrm{~kJ} \cdot \mathrm{mol}^{-1}$ and $26 \mathrm{~h}$ at $298 \mathrm{~K}$, respectively (Figure S9).

The effectiveness and selectivity of the photo-isomerization and the parameters of the thermal conversion step in solution coincide with those of other first-generation molecular motors with amide linkers, ${ }^{68}$ showing that the bulky groups do not interfere with the motor rotation. This lack of steric hindrance is due to the relative flexibility of the amide linker, which allows both bulky groups to point away from each other.

In contrast to solution, in the solid state, both the photochemical and thermal isomerization pathways of $\mathbf{1}$ are blocked. Irradiating the powder of trans-stable 1 extensively over time did not yield cis-unstable 1 indicated by ${ }^{1} \mathrm{H}$ NMR (Figure S10). Also, solid cis-unstable 1 did not undergo THI (Figure S11). It appears that the tight packing in the solid state does not create enough space for conformational rearrangement. An intermediate state of confinement can be found in the bowl-shaped aggregates. Use of solvent/non-solvent mixing $\left(\mathrm{THF}-\mathrm{H}_{2} \mathrm{O}\right)$ in different ratios gave control over the confinement of motor $\mathbf{1}$ and concomitantly its fluorescence and rotary behavior. With increasing $\varphi_{\mathrm{w}}$, the concentration of the motor into bowl-shaped aggregates resulted in aggregationinduced emission (AIE) upon UV irradiation (Figure 7). ${ }^{69-73}$ 
A
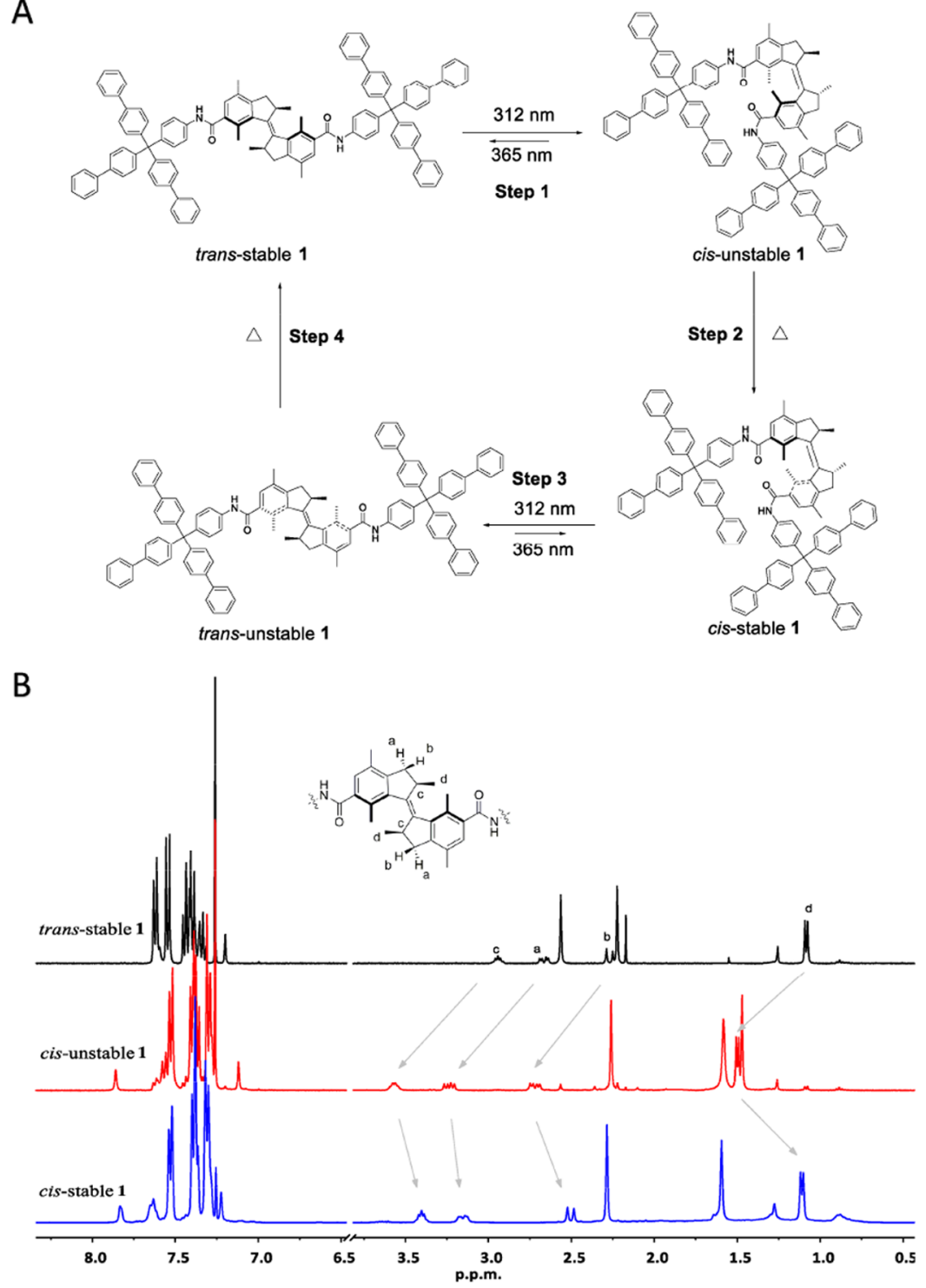

C

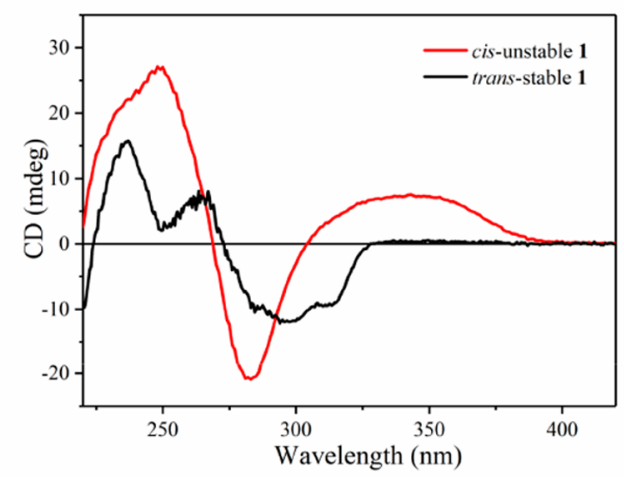

Figure 6. Rotary behavior of the bulky molecular motor 1 in solution. (A) The schematic $360^{\circ}$ rotary cycle of molecular motor 1 starting from trans-stable 1. (B) ${ }^{1} \mathrm{H}$ NMR spectra $\left(\mathrm{CDCl}_{3}\right)$ of trans-stable $\mathbf{1}$, cis-stable 1 by irradiating trans-stable 1 with $312 \mathrm{~nm} \mathrm{UV} \mathrm{light,} \mathrm{and} \mathrm{the} \mathrm{subsequent}$ cis-stable 1 by heating at $50{ }^{\circ} \mathrm{C}$ for $12 \mathrm{~h}$. (C) CD spectra of trans-stable and cis-unstable isomers of 1 in THF. $[1]=10^{-5} \mathrm{M}$.

As shown in Figure 7A, trans-stable 1 displays no fluorescence in pure THF. When $\varphi_{\mathrm{w}}$ is increased to $\sim 60 \%$, bowl-shaped particles are formed (Figure 3) and the motor shows fluorescence, which intensifies as the water concen- tration increases. Similar AIE behavior is also displayed by the other isomers (Figure 7B). The fluorescence quantum yield is moderate: for cis-unstable, it increases from $0.3 \%$ at $\varphi_{\mathrm{w}}=0$ to $2.7 \%$ at $\varphi_{\mathrm{w}}=90 \%$ (Figure S12). Due to the distinct electronic 

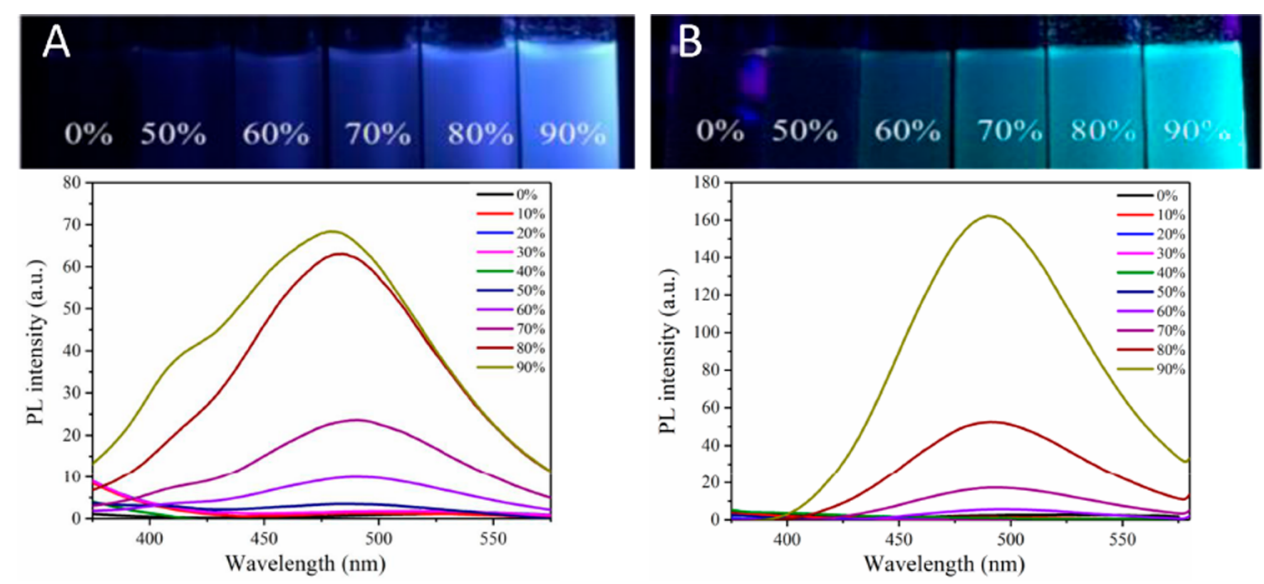

Figure 7. Fluorescence images and spectra of (A) trans-stable $\mathbf{1}$ and (B) cis-unstable $\mathbf{1}$ in aggregates formed in THF-water with different water fraction $\varphi_{\mathrm{w}}$. The total concentration of 1 in the mixtures was maintained at $10^{-4} \mathrm{M}$ and $\lambda_{\mathrm{ex}}=312 \mathrm{~nm}$.

structures of the isomers, the purplish blue fluorescence of trans-stable $1\left(\lambda_{\max }=482 \mathrm{~nm}\right)$ shifts to greenish blue for the cis-unstable $1\left(\lambda_{\max }=490 \mathrm{~nm}\right)$ (Figures 7, S13, and S14). Electron microscopy data indicate no difference in morphology or size of the aggregates of the isomers of 1 .

To determine the photochemical isomerization process in $s i t u$ in the aggregates, the fluorescence of trans-stable 1 in $\varphi_{\mathrm{w}}=$ $90 \%$ was monitored while irradiating with $312 \mathrm{~nm}$ UV light (Figure 8A). While morphologically the aggregates do not change, the broad emission band gradually becomes narrower accompanied by a disappearance of the shoulder at $415 \mathrm{~nm}$ and a slight red-shift of the whole spectrum, which indicates the formation of cis-unstable 1 . The clear isosbestic point demonstrates that there is a selective isomerization process

\section{A}

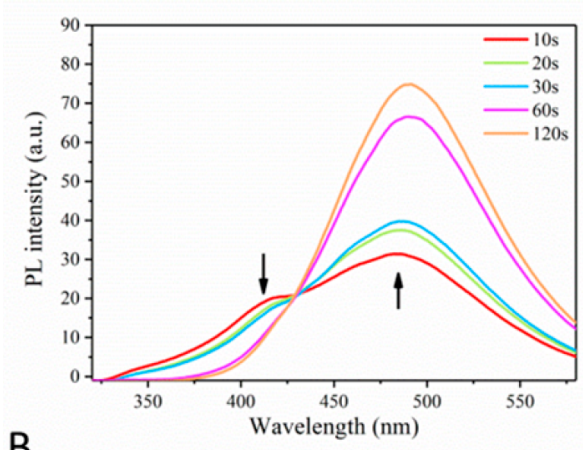

B

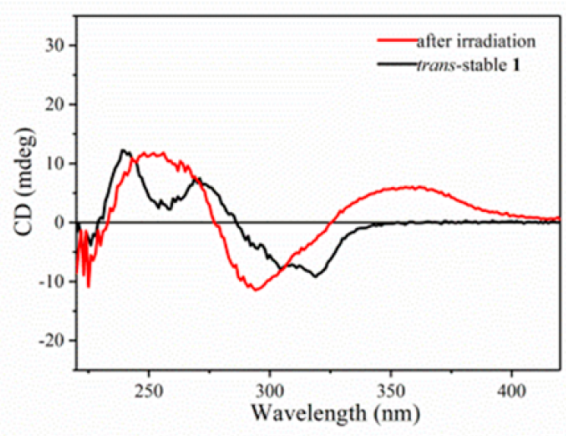

Figure 8. (A) Fluorescence spectral change on irradiating aggregated trans-stable 1 in THF-water at $\varphi_{\mathrm{w}}=90 \%$, and (B) its corresponding CD spectral change. $[1]=10^{-5} \mathrm{M}$. during the irradiation. The photoisomerization process is also confirmed by $\mathrm{CD}$ analysis using enantiopure compound $\mathbf{1}$ (Figure $8 \mathrm{~B}$ ). The aggregates of trans-stable 1 are $\mathrm{CD}$-silent at $>350 \mathrm{~nm}$; however, a positive Cotton effect emerges at $362 \mathrm{~nm}$ upon irradiation with UV light, which confirms the generation of cis-unstable 1. In contrast to the CD spectra in solution, there is a red-shift of the Cotton effect in the aggregated state which is attributed to light scattering of the aggregates. To confirm the photochemical conversion, ${ }^{1} \mathrm{H}$ NMR analysis was used. After reaching its PSS at $\varphi_{\mathrm{w}}=90 \%$, a ratio of $33 \%$ transstable 1 and $67 \%$ cis-unstable 1 was established (Figure S15A). A lower $\varphi_{\mathrm{w}}$ led to faster formation of the PSS as well as higher conversion, e.g., at $\varphi_{\mathrm{w}}=60 \%$ the PSS ratio (cis-unstable:transstable $=95: 5$ ) is similar to that in solution (Figure S16A).

To analyze the THI of $\mathbf{1}$ in confined space compared to the isomerization in solution, trans-stable 1 was irradiated to cisunstable 1 in THF and subsequently mixed with water $\left(\varphi_{\mathrm{w}}=\right.$ $90 \%)$ to obtain the aggregates. In contrast to its thermal behavior in solution, cis-unstable $\mathbf{1}$ in the aggregated state was unable to undergo THI (Figure 6A, step 2). Even in the case of prolonged heating, ${ }^{1} \mathrm{H}$ NMR analysis showed there was mainly cis-unstable 1 (Figure S17). While the photochemical isomerization to cis-unstable isomer 1 is uncompromised (Figure 6A step 1, Figure S15A), the absence of cis-stable $\mathbf{1}$ indicates that at $\varphi_{\mathrm{w}}=90 \%$ the THI step is blocked by the confined space of the nanospheres.

Further study of the thermal relaxation inside the aggregates uncovered an intriguing alternative thermal pathway for in situ generated cis-unstable 1 . To our surprise, the proportion of trans-stable 1 increased after heating while there was no presence of cis-stable 1 (Figure S15B). To elucidate this phenomenon, quantitative ${ }^{1} \mathrm{H}$ NMR analysis was conducted by adding an internal reference compound 6 (Figure 9, structure of 6 see SI, Scheme 1). Before thermal relaxation, the ratio of cis-unstable 1 and trans-stable $\mathbf{1}$ is 1:0.79. After heating, an increased amount of trans-stable $\mathbf{1}$ is observed accompanied by a corresponding decrease of cis-unstable $\mathbf{1}$, changing the ratio to $0.72: 1.07$. While the THI step is blocked (Figure 6A, step 2 ), in situ generated cis-unstable isomers can undergo a thermal cis-trans isomerization (Figure 6A, step 1 reversed). This process has usually a much higher energy barrier in the molecularly dissolved state than the THI process. Remarkably, in contrast to the aggregates at $\varphi_{\mathrm{w}}=90 \%$, in the systems at $\varphi_{\mathrm{w}}$ $=60 \%$, THI does occur (Figure S16B). 

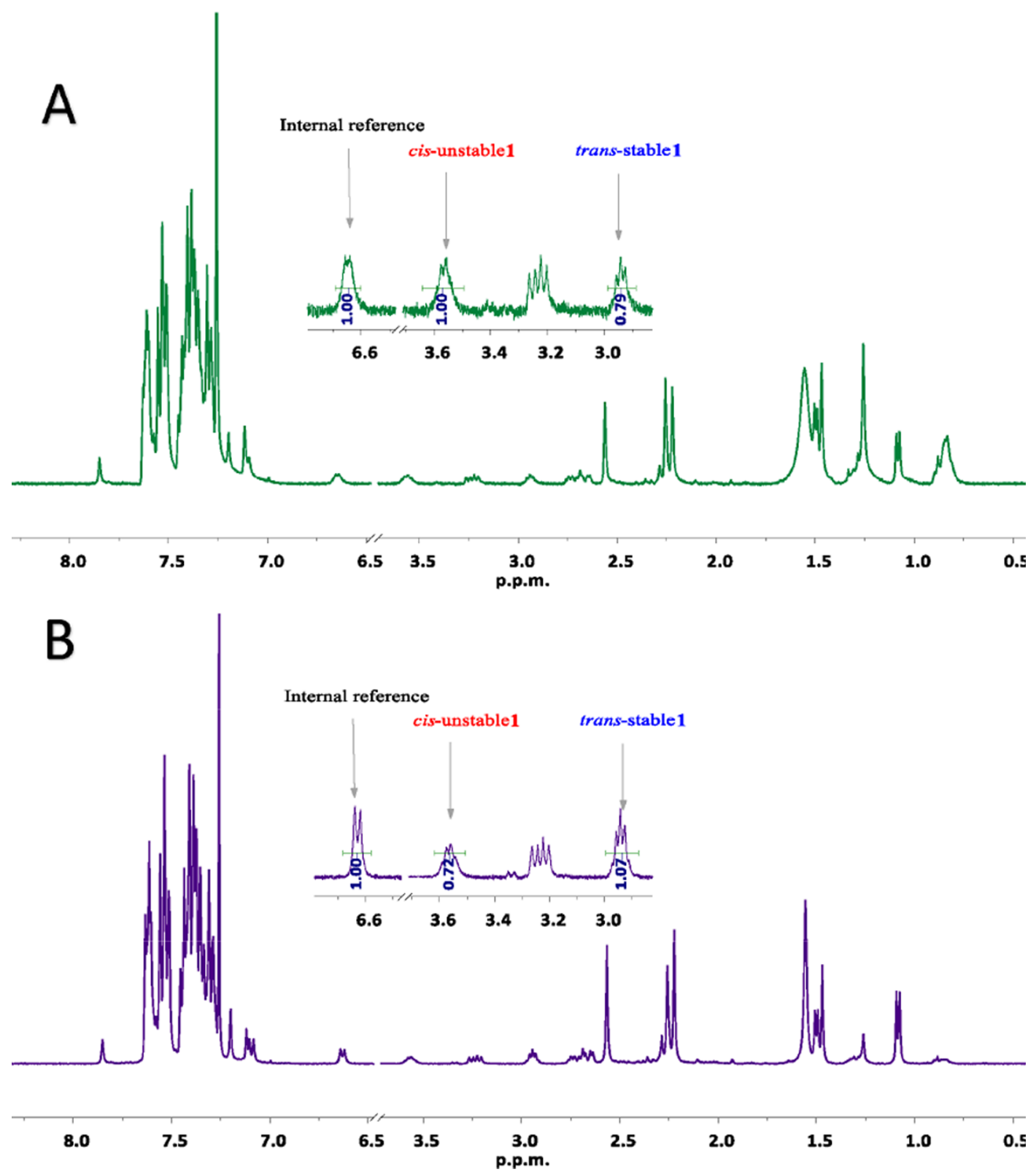

Figure 9. ${ }^{1} \mathrm{H}$ NMR spectral change of the thermal relaxation in the aggregates. The mixture was prepared by adding water $(54 \mathrm{~mL})$ to trans-stable 1 $\left(6 \times 10^{-3} \mathrm{mmol}\right)$ and compound $6\left(3 \times 10^{-3} \mathrm{mmol}\right.$, also see SI Scheme 1$)$ in THF $(6 \mathrm{~mL})$, followed by irradiating it with $312 \mathrm{~nm}$ UV light for 1 h. (A) Half of the mixture was separated and centrifuged; (B) the other half was heated at $50{ }^{\circ} \mathrm{C}$ for $48 \mathrm{~h}$ and then centrifuged.

Apparently, the increased confinement at high $\varphi_{\mathrm{w}}$ changes the motor behavior and allows the rotary motor to switch back to regenerate trans-stable 1 . These results demonstrate an intriguing discovery: i.e., the ratio of solvent and cosolvent and the extent to which nanosphere confinement takes place can dictate forward versus backward motion in a light-driven rotary motor.

\section{CONCLUSIONS}

In summary, based on the discovery of bowl-shaped aggregates obtained from a molecular motor, we showed how and why weak amphiphiles and hydrophobic molecules can assemble into bowl-shaped particles under the influence of solvent mixing. We demonstrate that the spheres are neither hollow, vesicular, nor micellar. The dense spheres with a fluid interior and a glass-like shell can be made from various materials, which suggests that this aggregation behavior should be termed not self-assembly, but solvent-driven assembly. The aggregates can shrink and swell reversibly upon addition of non-solvent or solvent, respectively, giving control over the extent of confinement inside the spheres.

We use this aggregation phenomenon to control the rotary behavior of the bulky molecular motor $\mathbf{1}$ by influencing the photochemical and thermal isomerization processes. Upon confinement, the energy barriers that determine the rotary motion of the motor change, blocking cis-unstable 1's forward isomerization, while allowing a thermal backward isomerization, i.e., reversal of cis-unstable to trans-stable state. In the aggregated state, molecular motor 1 exhibits also photoswitchable AIE behavior as the fluorescence can switch from purplish blue to greenish blue.

We expect that the elucidation of the actual nature of bowlshaped aggregates and the proposed mechanism of their formation will open the door to a wide range of applications 
taking advantage of their controllable size and fluid interior, which allow loading, compartmentalization, and confinement. The remarkable control of forward and backward rotary motion in light-driven motors by simply changing solvent ratios and thus extent of confinement is a fine example of several fascinating opportunities ahead of us for tuning of dynamic function at the nanoscale.

\section{ASSOCIATED CONTENT}

\section{S Supporting Information}

The Supporting Information is available free of charge on the ACS Publications website at DOI: 10.1021/jacs.8b03045.

Materials and methods, as well as supplementary data (PDF)

Movie S1 (AVI)

\section{AUTHOR INFORMATION}

\section{Corresponding Authors}

*b.l.feringa@rug.nl

*m.c.a.stuart@rug.nl

\section{ORCID}

Linda E. Franken: 0000-0002-3040-8192

Yuchen Wei: 0000-0003-2990-8226

Jiawen Chen: 0000-0002-0251-8976

Depeng Zhao: 0000-0002-3058-9958

Marc C. A. Stuart: 0000-0003-0667-6338

Ben L. Feringa: 0000-0003-0588-8435

\section{Present Addresses}

"L.E.F.: Department of Structural Cell Biology of Viruses, Heinrich Pette Institute, Leibniz Institute for Experimental Virology, Centre for Structural Systems Biology, Notkestraße 85, 22607 Hamburg, Germany

\#D.Z.: School of Pharmaceutical Sciences, Sun Yat-sen University, Guangzhou 510006, China

\section{Author Contributions}

${ }^{\perp}$ L.E.F. and Y.W. contributed equally.

\section{Notes}

The authors declare no competing financial interest.

\section{ACKNOWLEDGMENTS}

This research is financed in part by the BioSolar Cells open innovation consortium, supported by the Dutch Ministry of Economic Affairs, Agriculture and Innovation (LEF). B.L.F. acknowledges The Netherlands Organization for Scientific Research (NOW-CW), the Royal Netherlands Academy of Arts and Sciences (KNAW), and the Ministry of Education Culture and Science (Gravitation program 024.601035) for funding. Our thanks to Dr. Anton Hofman for supplying us with the polymers (Figure 2), Prof. Dr. Katja Loos for valuable discussions, and Dr. Sander Wezenberg for discussion and proofreading the manuscript.

\section{REFERENCES}

(1) Allen, T. M.; Cullis, P. R. Adv. Drug Delivery Rev. 2013, 65, 36.

(2) Decher, G. Science 1997, 277, 1232.

(3) Grzelczak, M.; Vermant, J.; Furst, E. M.; Liz-Marzan, L. M. ACS Nano 2010, 4, 3591.

(4) Zeng, F.; Zimmerman, S. Chem. Rev. 1997, 97, 1681.

(5) Stuart, M. A. C.; Huck, W. T. S.; Genzer, J.; Mueller, M.; Ober, C.; Stamm, M.; Sukhorukov, G. B.; Szleifer, I.; Tsukruk, V. V.; Urban,
M.; Winnik, F.; Zauscher, S.; Luzinov, I.; Minko, S. Nat. Mater. 2010, 9, 101.

(6) Ganta, S.; Devalapally, H.; Shahiwala, A.; Amiji, M. J. Controlled Release 2008, 126, 187.

(7) Jochum, F. D.; Theato, P. Chem. Soc. Rev. 2013, 42, 7468.

(8) Mura, S.; Nicolas, J.; Couvreur, P. Nat. Mater. 2013, 12, 991.

(9) Schneider, J.; Pochan, D.; Ozbas, B.; Rajagopal, K.; Pakstis, L.; Kretsinger, J. J. Am. Chem. Soc. 2002, 124, 15030.

(10) Toohey, K. S.; Sottos, N. R.; Lewis, J. A.; Moore, J. S.; White, S. R. Nat. Mater. 2007, 6, 581.

(11) Cordier, P.; Tournilhac, F.; Soulie-Ziakovic, C.; Leibler, L. Nature 2008, 451, 977.

(12) Zhang, M.; Xu, D.; Yan, X.; Chen, J.; Dong, S.; Zheng, B.; Huang, F. Angew. Chem., Int. Ed. 2012, 51, 7011.

(13) Yokoyama, M.; Satoh, A.; Sakurai, Y.; Okano, T.; Matsumura, Y.; Kakizoe, T.; Kataoka, K. J. Controlled Release 1998, 55, 219.

(14) Yang, X.; Grailer, J. J.; Rowland, I. J.; Javadi, A.; Hurley, S. A.; Steeber, D. A.; Gong, S. Biomaterials 2010, 31, 9065.

(15) Nicolas, J.; Mura, S.; Brambilla, D.; Mackiewicz, N.; Couvreur, P. Chem. Soc. Rev. 2013, 42, 1147.

(16) Torchilin, V. P. Pharm. Res. 2007, 24, 2333.

(17) Leenders, S. H. A. M.; Gramage-Doria, R.; de Bruin, B.; Reek, J. N. H. Chem. Soc. Rev. 2015, 44, 433.

(18) Yoshizawa, M.; Tamura, M.; Fujita, M. Science 2006, 312, 251.

(19) Zhao, H.; Sen, S.; Udayabhaskararao, T.; Sawczyk, M.; Kucanda, K.; Manna, D.; Kundu, P. K.; Lee, J.; Kral, P.; Klajn, R. Nat. Nanotechnol. 2015, 11, 82.

(20) Chen, J.; Leung, F. K.; Stuart, M. C. A.; Kajitani, T.; Fukushima, T.; van der Giessen, E.; Feringa, B. L. Nat. Chem. 2017, $10,132$.

(21) Goujon, A.; Du, G.; Moulin, E.; Fuks, G.; Maaloum, M.; Buhler, E.; Giuseppone, N. Angew. Chem., Int. Ed. 2016, 55, 703.

(22) Park, C.; Yoon, J.; Thomas, E. Polymer 2003, 44, 6725.

(23) Groeschel, A. H.; Schacher, F. H.; Schmalz, H.; Borisov, O. V.; Zhulina, E. B.; Walther, A.; Mueller, A. H. E. Nat. Commun. 2012, 3, 710.

(24) Mai, Y.; Eisenberg, A. Chem. Soc. Rev. 2012, 41, 5969.

(25) Hartgerink, J.; Beniash, E.; Stupp, S. Proc. Natl. Acad. Sci. U. S. A. 2002, 99, 5133.

(26) Berzin, F.; Vergnes, B.; Delamare, L. J. Appl. Polym. Sci. 2001, $80,1243$.

(27) Tzoganakis, C.; Vlachopoulos, J.; Hamielec, A. Polym. Eng. Sci. 1988, 28, 170.

(28) Brizard, A.; Stuart, M.; van Bommel, K.; Friggeri, A.; de Jong, M.; van Esch, J. Angew. Chem., Int. Ed. 2008, 47, 2063.

(29) Denisov, I.; Grinkova, Y.; Lazarides, A.; Sligar, S. J. Am. Chem. Soc. 2004, 126, 3477.

(30) Hyuk Im, S.; Jeong, U.; Xia, Y. Nat. Mater. 2005, 4, 671.

(31) Maity, S.; Jana, P.; Maity, S. K.; Haldar, D. Langmuir 2011, 27, 3835.

(32) Yang, Z.; Wang, X.; Yang, Y.; Liao, Y.; Wei, Y.; Xie, X. Langmuir 2010, 26, 9386.

(33) Liu, X.; Liu, J.; Jiang, M. Macromol. Rapid Commun. 2009, 30, 892.

(34) Wang, J.; Kuang, M.; Duan, H.; Chen, D.; Jiang, M. Eur. Phys. J. E: Soft Matter Biol. Phys. 2004, 15, 211.

(35) Li, G.; Du, F.; Wang, H.; Bai, R. React. Funct. Polym. 2014, 75, 75 .

(36) Riegel, I.; Eisenberg, A.; Petzhold, C.; Samios, D. Langmuir 2002, 18, 3358

(37) Riegel, I.; de Bittencourt, F.; Terrau, O.; Eisenberg, A.; Petzhold, C.; Samios, D. Pure Appl. Chem. 2004, 76, 123.

(38) Yao, W.; Li, Y.; Feng, C.; Lu, G.; Huang, X. Polym. Chem. 2014, 5,6334 .

(39) Jiang, X.; Jiang, X.; Lu, G.; Feng, C.; Huang, X. Polym. Chem. 2014, 5, 4915.

(40) Jin, Y.; Song, L.; Wang, D.; Qiu, F.; Yan, D.; Zhu, B.; Zhu, X. Soft Matter 2012, 8, 10017.

(41) Sun, Y.; Liu, W. J. Polym. Res. 2012, 19, 9768. 
(42) Mitra, A.; Panda, D. K.; Corson, L. J.; Saha, S. Chem. Commun. 2013, 49, 4601.

(43) Vriezema, D. M.; Hoogboom, J.; Velonia, K.; Takazawa, K.; Christianen, P. C. M.; Maan, J. C.; Rowan, A. E.; Nolte, R. J. M. Angew. Chem., Int. Ed. 2003, 42, 772.

(44) He, Q.; Ao, Y.; Huang, Z.; Wang, D. Angew. Chem., Int. Ed. 2015, 54, 11785.

(45) Vriezema, D. M.; Garcia, P. M. L.; Oltra, N. S.; Hatzakis, N. S.; Kuiper, S. M.; Nolte, R. J. M.; Rowan, A. E.; van Hest, J. C. M. Angew. Chem., Int. Ed. 2007, 46, 7378.

(46) van Dongen, S. F. M.; Nallani, M.; Cornelissen, J. L. L. M.; Nolte, R. J. M.; van Hest, J. C. M. Chem. - Eur. J. 2009, 15, 1107.

(47) Alfonso, I.; Bru, M.; Burguete, M. I.; Garcia-Verdugo, E.; Luis, S. V. Chem. - Eur. J. 2010, 16, 1246.

(48) Tao, W.; Liu, Y.; Jiang, B.; Yu, S.; Huang, W.; Zhou, Y.; Yan, D. J. Am. Chem. Soc. 2012, 134, 762.

(49) Choucair, A.; Eisenberg, A. Eur. Phys. J. E: Soft Matter Biol. Phys. 2003, 10, 37.

(50) Yu, Y.; Eisenberg, A. J. Am. Chem. Soc. 1997, 119, 8383.

(51) Shen, H.; Eisenberg, A. J. Phys. Chem. B 1999, 103, 9473.

(52) Zhang, L.; Eisenberg, A. Science 1995, 268, 1728.

(53) Whitesides, G.; Grzybowski, B. Science 2002, 295, 2418.

(54) Koumura, N.; Zijlstra, R.; van Delden, R.; Harada, N.; Feringa, B. Nature 1999, 401, 152.

(55) Koumura, N.; Geertsema, E.; van Gelder, M.; Meetsma, A.; Feringa, B. J. Am. Chem. Soc. 2002, 124, 5037.

(56) van Delden, R.; ter Wiel, M.; Pollard, M.; Vicario, J.; Koumura, N.; Feringa, B. Nature 2005, 437, 1337.

(57) Chen, J.; Kistemaker, J. C. M.; Robertus, J.; Feringa, B. L. J. Am. Chem. Soc. 2014, 136, 14924.

(58) Kistemaker, J. C. M.; Lubbe, A. S.; Bloemsma, E. A.; Feringa, B. L. ChemPhysChem 2016, 17, 1819.

(59) Smith, S. O. Annu. Rev. Biophys. 2010, 39, 309.

(60) Becker, R.; Freedman, K. J. Am. Chem. Soc. 1985, 107, 1477.

(61) Benard, S.; Yu, P. Adv. Mater. 2000, 12, 48.

(62) Bushuyev, O. S.; Tomberg, A.; Friscic, T.; Barrett, C. J. J. Am. Chem. Soc. 2013, 135, 12556.

(63) Harada, J.; Kawazoe, Y.; Ogawa, K. Chem. Commun. 2010, 46, 2593.

(64) Kobatake, S.; Takami, S.; Muto, H.; Ishikawa, T.; Irie, M. Nature 2007, 446, 778.

(65) Naito, T.; Horie, K.; Mita, I. Macromolecules 1991, 24, 2907.

(66) Franken, L. E.; Boekema, E. J.; Stuart, M. C. A. Adv. Sci. 2017, $4,1600476$.

(67) Couchman, P.; Karasz, F. Macromolecules 1978, 11, 117.

(68) Zhao, D.; Neubauer, T. M.; Feringa, B. L. Nat. Commun. 2015, 6, 6652 .

(69) Mei, J.; Hong, Y.; Lam, J. W. Y.; Qin, A.; Tang, Y.; Tang, B. Z. Adv. Mater. 2014, 26, 5429.

(70) Wang, J.; Mei, J.; Hu, R.; Sun, J. Z.; Qin, A.; Tang, B. Z. J. Am. Chem. Soc. 2012, 134, 9956.

(71) Luo, J.; Xie, Z.; Lam, J.; Cheng, L.; Chen, H.; Qiu, C.; Kwok, H.; Zhan, X.; Liu, Y.; Zhu, D.; Tang, B. Chem. Commun. 2001, 18, 1740.

(72) Zhao, Z.; He, B.; Tang, B. Z. Chem. Sci. 2015, 6, 5347.

(73) Mei, J.; Leung, N. L. C.; Kwok, R. T. K.; Lam, J. W. Y.; Tang, B. Z. Chem. Rev. 2015, 115, 11718. 